\title{
Effect of heating and cooling of photogenerated electron-hole plasma in optically pumped graphene on population inversion.
}

\author{
V. Ryzhii ${ }^{1,4}$, M. Ryzhii ${ }^{1,4}$, V. Mitin ${ }^{2}$, A. Satou ${ }^{3,4}$, and T. Otsuji ${ }^{3,4}$ \\ 1 Computational Nanoelectronics Laboratory, \\ University of Aizu, Aizu-Wakamatsu, 965-8580, Japan \\ 2 Department of Electrical Engineering, \\ University at Buffalo, Buffalo, NY 1460-1920, U.S.A. \\ 3 Research Institute for Electrical Communication, \\ Tohoku University, Sendai, 980-8577, Japan \\ 4 Japan Science and Technology Agency, \\ CREST, Tokyo 10\%-0075, Japan
}

\begin{abstract}
We study the characteristics of photogenerated electron-hole plasma in optically pumped graphene layers at elevated (room) temperatures when the interband and intraband processes of emission and absorption of optical phonons play a crucial role. The electron-hole plasma heating and cooling as well as the effect of nonequilibrium optical phonons are taken into account. The dependences of the quasi-Fermi energy and effective temperature of optically pumped graphene layers on the intensity of pumping radiation are calculated. The variation of the frequency dependences dynamic conductivity with increasing pumping intensity as well as the conditions when this conductivity becomes negative in a certain range of frequencies are considered. The effects under consideration can markedly influence the achievement of the negative dynamic conductivity in optically pumped graphene layers associated with the population inversion and, hence, the realization graphene-based terahertz and infrared lasers operating at room temperatures.
\end{abstract}

\section{INTRODUCTION}

The gapless energy spectrum of graphene layers (GLs) 1] provides an opportunity to create devices utilizing the interband transitions in a wide spectral range of radiation. As shown [2] (see also Refs. [3 5]), under optical or electrical pumping, the interband population inversion can be realized in GLs. Due to fairly high quantum efficiency of the interband transitions in GL [6, 7] and particularly in multiple-GL structures [8, 9], the interband contribution to the real part of the dynamic conductivity can surpass that associated with the intraband (Drude) absorption [2], so that the GL net dynamic conductivity is negative in a certain range of signal frequencies. This opens up the prospects of achieving of lasing of the terahertz $(\mathrm{THz})$ and infrared (IR) radiation in different structures on the base of single- and multipleGLs [10 12]. In optically pumped GLs, a marked portion of the absorbed optical energy can go to the electron and hole heating. As demonstrated [5, 13 15], the electron and hole heating hinders the realization of population inversion and negativity of dynamic conductivity. At elevated (room) temperatures, in contrast to the low temperature situation [13], the interband transitions associated with optical phonons can be the dominant mechanism of the recombination [16]. The energy relaxation of electrons and holes in GLs can also be mainly due to interaction with optical phonons [17. In such a case, the optical photons emitted by the photogenerated electrons and holes can accumulate in GL, so that the optical phonon system is heated as well. This can lead to the optical photons reabsorption accompanied with the intraband electron and hole transitions. The deviation of the optical phonon system from equilibrium can also affect the properties of GL, as it occurs in carbon nanotubes (see for instance Refs. [18 24]), in particular, affect the interband generation-recombination processes. Increase of the number of nonequilibrium phonons result in the increase of stimulated optical phonon emission and may lead to the generation of coherent phonons in graphene like it was discussed for a different systems [25 27]. Thus, the accounting for the optical phonon heating appears to be indispensable. However, it is important to stress that at certain values of the pumping radiation frequencies, cooling of the electron-hole plasma below the temperature of the lattice is possible.

In this paper, we study the effect of heating and cooling of the electron-hole plasma in optically pumped GLs at elevated temperatures, when the interaction with optical phonons is the main mechanism of the recombination and energy relaxation, on its characteristics, particularly on its dynamic conductivity and in the $\mathrm{THz}$ and IR frequency ranges. The deviation of the optical phonon system from equilibrium (optical phonon heating) is taken into account The obtained characteristics are crucial for the realization of $\mathrm{THz}$ and IR lasers [2] as well as different $\mathrm{THz}$ plasma wave devices [28, 29] on GL structures operating at room temperatures and can be used for the device optimization.

\section{MODEL AND THE PERTINENT EQUATIONS}

We consider the interband phogeneration of electrons and holes by optical radiation with the photon energy 
$\hbar \Omega$ (optical pumping), where $\hbar$ is the reduced Planck constant. After the photogeneration of an electron and a hole with the energy exceeding the optical phonon energy $\hbar \omega_{0} \sim 200 \mathrm{meV}$, their behavior can correspond to the following tracks:

(a) If the characteristic time, $\tau_{c c}$, of inter-carrier scattering (electron-electron, electron-hole, and hole-hole) is much larger than the characteristic time, $\tau_{0}$ of spontaneous emission of optical phonon just photogenerated carriers can manage to emit cascades of optical phonons. The number of optical phonons $K$ in such a cascade is determined by the ration $\Omega / \omega_{0}$. Due to the symmetry of the energy spectrum, the photogenerated electrons and holes emit equal number $(K)$ of phonons. This cascade is followed by the electron and hole "fermisation" and recombination 2], so that the electron and hole distribution function becomes equal to $f=\left\{\exp \left[\left(\varepsilon-\varepsilon_{F}\right) / T\right]+1\right\}^{-1}$. Here $\varepsilon=v_{W} p$ is the energy of electrons and holes with the momentum $p, v_{W}=10^{8} \mathrm{~cm} / \mathrm{s}$ is the characteristic velocity of the GL energy spectrum, $\varepsilon_{F}$ is the quasi-Fermi energy, and $T$ is the effective temperature (in the energy units). The quasi Fermi energy of the electron-hole plasma in the situation in question $\varepsilon_{F}$ and its effective temperature $T$ are determined by the rates of photogeneration, and recombination, as well as the rates intraband and interband energy relaxation. These parameters are determined by the energy $\varepsilon_{0}$ which is supplied to the electron-hole plasma in each act of the photogeneration. In the case in question, $\varepsilon_{0}=\hbar \Omega_{0}=\hbar \Omega-2 K \hbar \omega_{0}$. Generally, $\varepsilon_{F}$ and $T$ are not equal to their equilibrium values, i.e., $\varepsilon_{F} \neq 0$ and $T \neq T_{0}$;

(b) If the inter-carrier scattering time is the shortest one $\left(\tau_{c c} \ll \tau_{0}\right)$, just photogenerated electrons and holes are immediately "fermilised". In this case, all the energy of photogenerated carriers goes to the electron-hole plasma, so that $\varepsilon_{0}=\hbar \Omega$;

(c) At relatively low energies of photons $\left(\hbar \Omega<2 \hbar \omega_{0}\right)$, the situation is similar to the previous case with $\varepsilon_{0}=\hbar \Omega$, but for rather arbitrary relationships between the characteristic times of inter-carrier and optical phonon scattering. This case corresponds, in particular, to optical pumping by $\mathrm{CO}_{2}$ or quantum-cascade lasers;

(d) When $\hbar \Omega>\hbar \omega_{0}$ (or $\hbar \Omega \gg \hbar \omega_{0}$ ), and the characteristic times of inter-carrier and optical phonon scattering are of the same order of magnitude $\left(\operatorname{tau}_{c c} \sim \tau_{0}\right)$, the pattern of the energy relaxation of photogenerated carriers can be fairly complex. Nevertheless, even in this case, on can introduce the energy $\varepsilon_{0}=\hbar \Omega_{0}$, which, however, is determined not only by the ration of $\hbar \Omega$ and $\hbar \omega_{0}$, but also by the ratio of the characteristic scattering times. Naturally, $\hbar \Omega-2 K \hbar \omega_{0}<\varepsilon_{0}<\hbar \Omega$. In this case, the quantity $\varepsilon_{0}$ (or $\Omega_{0}$ ) is a phenomenological parameter, which determines the fractions of the absorbed photon energy going to directly to the electronhole plasma and to the optical phonon system. For a rough estimate, the following simple formula might be used: $\hbar \Omega_{0}=\hbar \Omega-2 K \hbar \omega_{0} /\left[1+K \tau_{0} / \tau_{c c}\right]$, which yields the above values of $\hbar \Omega_{0}$ in the limiting cases (a) and (b).
Introducing parameter $\varepsilon_{0}$ (or $\left.\Omega_{0}\right)$, one can consider all the abovementioned tracks in the framework of the same treatment.

In the situation under consideration, the optical phonon system can also be far from equilibrium, so that the distribution function of optical phonons $\mathcal{N}_{0}$ can markedly deviated from its equilibrium value $\mathcal{N}_{0}^{\text {eq }}=$ $\left[\exp \left(\hbar \omega_{0} / T_{0}\right)-1\right]^{-1}$. The latter can lead to an effective reabsorption of optical phonons by electrons and holes with a significant increase in their energies. Although at low temperatures, the energy relaxation and recombination of electrons and holes are associated with the interaction with acoustic phonons and radiative processes, respectively [13], in the case of elevated temperatures (say, room temperatures) and pumping intensities, the energy relaxation and recombination is assumed to be due to the interactions with optical phonons (both inraband and interband).

The quasi-Fermi energy of the electron-hole plasma $\varepsilon_{F}$, its effective temperature $T$, and the number of optical phonons $\mathcal{N}_{0}$ obey the equations

$$
\begin{gathered}
R_{0}^{\text {inter }}=G_{\Omega}, \\
\hbar \omega_{0}\left(R_{0}^{\text {inter }}+R_{0}^{\text {intra }}\right)=G_{\Omega_{0}} \hbar \Omega_{0}, \\
\hbar \omega_{0} R^{\text {decay }}=\hbar \Omega G_{\Omega},
\end{gathered}
$$

governing the balance of the electron-hole pairs, the electron-hole plasma energy balance, respectively, as well as the optical phonon balance. Here, $R_{0}^{\text {inter }}, R_{0}^{\text {intra }}$, and $R^{\text {decay }}$ are the rates of the pertinent processes. Equation (3) explicitly takes into account that all the energy received by the system from optical pumping goes eventually to the thermostat (to the contacts via acoustic phonons). The optical generation rate is given by

$$
G_{\Omega}=\pi \alpha \tanh \left(\frac{\hbar \Omega-2 \varepsilon_{F}}{4 T}\right) I .
$$

Here, $I$ is the photon flux of pumping radiation and $\alpha \simeq 1 / 137$ is the fine structure constant (so that the absorption coefficient is equal to $\pi \alpha \simeq 0.023$ ). The last factor in the right-hand side of Eq. (4) accounts for the limitation of the interband absorption associated with the Pauli blocking. In this case, $G_{\Omega} \simeq G_{\Omega_{0}} \simeq \pi \alpha I$. To provide an effective cascade pumping and avoid the fast direct transitions with the emission of an additional optical phonon, the following conditions should be satisfied:

$$
\varepsilon_{F}<\hbar \Omega_{0} / 2<\hbar \omega_{0}-\varepsilon_{F}
$$

\section{RATES OF THE PROCESSES UNDER CONSIDERATION}

For the terms $R_{0}^{\text {inter }}$ and $R_{0}^{\text {intra }}$, which describe the electron-hole recombination and generation processes 
and the intraband energy relaxation assisted by optical phonons, one can use the following simplified formulas:

$$
\begin{gathered}
R_{0}^{\text {inter }}=\frac{\Sigma_{0}}{\tau_{0}^{\text {inter }}}\left[\left(\mathcal{N}_{0}+1\right) \exp \left(\frac{2 \varepsilon_{F}-\hbar \omega_{0}}{T}\right)-\mathcal{N}_{0}\right] \\
R_{0}^{\text {intra }}=\frac{\Sigma_{0}}{\tau_{0}^{\text {intra }}}\left[\left(\mathcal{N}_{0}+1\right) \exp \left(-\frac{\hbar \omega_{0}}{T}\right)-\mathcal{N}_{0}\right]
\end{gathered}
$$

Here, $\Sigma_{0}=\pi\left(T_{0} / \hbar v_{W}\right)^{2} / 6$ is the equilibrium electron and hole density and $\tau_{0}^{\text {inter }} \propto \tau_{0}^{\text {intra }} \propto \tau_{0}$ are the times of the interband and intraband phonon-assisted processes. The latter quantities have been introduced in the present form for convenience

A difference in $\tau_{0}^{\text {inter }}$ and $\tau_{0}^{\text {intra }}$ is associated with the features of the density of states and with a difference between the optical phonon energy and the quasi-Fermi energy (see Appendix). In equilibrium, i.e., at $\mathcal{N}_{0}=\mathcal{N}_{0}^{e q}$, $\varepsilon_{F}=0$, and $T=T_{0}$, Eqs. (6) and (7) yield $R_{0}^{\text {inter }}=$ $R_{0}^{\text {intra }}=0$.

The rate of optical phonons decay due to the anharmonic contributions to the interatomic potential, resulting in the phonon-phonon scattering and in the decay of optical phonons into acoustic phonons and is assumed to be in the following form:

$$
R_{0}^{\text {decay }}=\frac{\Sigma_{0}\left(\mathcal{N}_{0}-\mathcal{N}_{0}^{e q}\right)}{\tau_{0}^{\text {decay }}}
$$

where $\tau_{0}^{\text {decay }}$ is the pertinent characteristic time. Considering high heat conductivity of GLs [30], the lattice temperature, i.e. the temperature of acoustic phonons, is assumed to be equal to the temperature of the contact $T_{0}$.

\section{CALCULATION OF THE EFFECTIVE} TEMPERATURE AND QUASI-FERMI ENERGY

Equations (1), (3), and (8) yield

$$
\mathcal{N}_{0}=\mathcal{N}_{0}^{e q}\left(1+\eta_{0}^{\text {decay }} \frac{I}{I_{0}}\right)
$$

Here

$$
I_{0}=\mathcal{N}_{0}^{e q} \frac{\omega_{0}}{\Omega} \frac{\Sigma_{0}}{\pi \alpha \tau_{0}^{\text {inter }}} \simeq \exp \left(-\frac{\hbar \omega_{0}}{T_{0}}\right) \frac{\omega_{0}}{\Omega} \frac{\Sigma_{0}}{\pi \alpha \tau_{0}^{\text {inter }}}
$$

is the characteristic photon flux and $\eta_{0}^{\text {decay }}=$ $\tau_{0}^{\text {decay }} / \tau_{0}^{\text {inter }}$. As follows from Eq. (9), at very large pumping intensities, $\mathcal{N}_{0}$ can markedly exceed unity. We shall not consider the range of such intensities. Therefore, in the following we limit our consideration by not too strong pumping assuming that $\eta_{0}^{\text {decay }} I / I_{0} \ll$ $\exp \left(\hbar \omega_{0} / T_{0}\right)$, i.e., $I \ll I_{0} \exp \left(\hbar \omega_{0} / T_{0}\right) / \eta_{0}^{\text {decay }}=I_{0}^{\infty}$.

Using Eqs. (1), (2), (5), and (7) with Eq. (9), we arrive at the following equations for the effective temperature $T$ and the quasi-Fermi energy $\varepsilon_{F}$ (for not too strong pumping intensities):

$$
\begin{aligned}
& \exp \left(\frac{2 \varepsilon_{F}-\hbar \omega_{0}}{k_{B} T}\right)=\frac{1+\left(\eta_{0}^{\text {decay }}+1\right) \frac{I}{I_{0}}}{\exp \left(\frac{\hbar \omega_{0}}{T_{0}}\right)+1+\eta_{0}^{\text {decay }} \frac{I}{I_{0}}} \\
& \simeq \exp \left(-\frac{\hbar \omega_{0}}{T_{0}}\right)\left[1+\left(\eta_{0}^{\text {decay }}+1\right) \frac{I}{I_{0}}\right]
\end{aligned}
$$

$$
\begin{aligned}
& \exp \left(-\frac{\hbar \omega_{0}}{k_{B} T}\right)=\frac{1+\left[\eta_{0}^{\text {decay }}+\eta_{0}\left(\frac{\Omega_{0}}{\omega_{0}}-1\right)\right] \frac{I}{I_{0}}}{\exp \left(\frac{\hbar \omega_{0}}{T_{0}}\right)+1+\eta_{0}^{\text {decay }} \frac{I}{I_{0}}} \\
& \simeq \exp \left(-\frac{\hbar \omega_{0}}{T_{0}}\right)\left\{1+\left[\eta_{0}^{\text {decay }}+\eta_{0}\left(\frac{\Omega_{0}}{\omega_{0}}-1\right)\right] \frac{I}{I_{0}}\right\},
\end{aligned}
$$

$$
\exp \left(\frac{2 \varepsilon_{F}}{T}\right)=\frac{1+\left(\eta_{0}^{\text {decay }}+1\right) \frac{I}{I_{0}}}{1+\left[\eta_{0}^{\text {decay }}+\eta_{0}\left(\frac{\Omega_{0}}{\omega_{0}}-1\right)\right] \frac{I}{I_{0}}}
$$

Here $\eta_{0}=\tau_{0}^{\text {intra }} / \tau_{0}^{\text {inter }}$.

Equations (11) and (12) yield

$$
T \simeq \frac{T_{0}}{1-\frac{T_{0}}{\hbar \omega_{0}} \ln \left\{1+\left[\eta_{0}^{\text {decay }}+\eta_{0}\left(\frac{\Omega_{0}}{\omega_{0}}-1\right)\right] \frac{I}{I_{0}}\right\}}
$$




$$
\varepsilon_{F} \simeq \frac{T_{0}}{2} \frac{\ln \left\{\frac{1+\left(\eta_{0}^{\text {decay }}+1\right) \frac{I}{I_{0}}}{1+\left[\eta_{0}^{\text {decay }}+\eta_{0}\left(\frac{\Omega_{0}}{\omega_{0}}-1\right)\right] \frac{I}{I_{0}}}\right\}}{1-\frac{T_{0}}{\hbar \omega_{0}} \ln \left\{1+\left[\eta_{0}^{\text {decay }}+\eta_{0}\left(\frac{\Omega_{0}}{\omega_{0}}-1\right)\right] \frac{I}{I_{0}}\right\}} .
$$

As it should be, Eqs. (13) and (14) yield $T=T_{0}$ and $\varepsilon_{F}=0$ if $I=0$.

The dependence of parameter $\eta_{0}$ on $\varepsilon_{F}$ and $T$ complicates the solution of Eqs. (13) and (14) in wide ranges of pumping intensities, particularly, their analytical solution.

\section{SPECIAL AND LIMITING CASES}

\section{A. Special case $\Omega_{0} / \omega_{0}=1$.}

In a special case $\Omega_{0} / \omega_{0}=1$ (i.e., $\left.\Omega=(2 K+1) \omega_{0}\right)$, Eqs. (12) - (14) yield

$$
\begin{gathered}
T \simeq \frac{T_{0}}{1-\frac{T_{0}}{\hbar \omega_{0}} \ln \left(1+\eta_{0}^{\text {decay }} I / I_{0}\right)} \\
\simeq T_{0}+\frac{T_{0}^{2}}{\hbar \omega_{0}} \ln \left(1+\eta_{0}^{\text {decay }} I / I_{0}\right), \\
\varepsilon_{F} \simeq \frac{T_{0}}{2} \frac{\ln \left(1+\frac{I / I_{0}}{1+\eta_{0}^{\text {decay }} I / I_{0}}\right)}{\hbar \omega_{0}} \ln \left(1+\eta_{0}^{\text {decay }} I / I_{0}\right) \\
\simeq \frac{T_{0}}{2} \ln \left(1+\frac{I / I_{0}}{1+\eta_{0}^{\text {decay }} I / I_{0}}\right), \\
\exp \left(\frac{2 \varepsilon_{F}}{T}\right)=1+\frac{I / I_{0}}{1+\eta_{0}^{\text {decay }} I / I_{0}} .
\end{gathered}
$$

Thus, at $\Omega_{0} / \omega_{0}=1$, the effective temperature $T$ is an increasing (logarithmic) function of the pumping intensity $I$, although the increase in $T$ is rather slow due to a small factor $T_{0} / \hbar \omega_{0} \simeq 0.125$ in the last pre-logarithmic term in the right-hand side of Eq. (15). As follows from Eq. (16), the quasi-Fermi energy $\varepsilon_{F}$ logarithmically increases with $I$ and tends to the saturates with $\varepsilon_{F}^{\text {sat }}=\left(T_{0} / 2\right) \ln \left(1+1 / \eta_{0}^{\text {decay }}\right)$. The increase in $\varepsilon_{F}$ in the range of moderate $I / I_{0}\left(I / I_{0} \lesssim 1 / \eta_{\text {decay }}\right)$ is faster than that in $T$. One can also see that an increase in the decay time leads to slowing down the $\varepsilon_{F}-I$ dependence.

One can see that the variation of the effective temperature (the electron-hole plasma heating) is associate with the finiteness of the optical phonon decay time, i.e., with the optical phonon heating and the absorption of hot phonons by electrons and holes, while the contributions of the intraband and interband emission and absorption of optical phonons to the electron-hole plasma energy balance at $\Omega_{0} / \omega_{0}$ are compensated. It is instructive that in the case in question, the effective temperature of the optical phonon system $\Theta$, defined in such a way that $\mathcal{N}_{0}=\left[\exp \left(\hbar \omega_{0} / \Theta\right)-1\right]^{-1}$, exceeds the lattice temperature $T_{0}$ (optical phonon heating) and is equal to the effective temperature of the electron-hole plasma $T$. Indeed, using Eq. (9), we find (compare with Eq. (15))

$$
\Theta \simeq \frac{T_{0}}{1-\frac{T_{0}}{\hbar \omega_{0}} \ln \left(1+\eta_{0}^{\text {decay }} I / I_{0}\right)} .
$$

The fact that $\Theta=T>T_{0}$ implies that both electronhole plasma and optical phonon system are heated and they are in equilibrium with each other.

If $\hbar \Omega=1 \mathrm{eV}$, so that $K=2$, assuming that $T_{0}=$ $300 \mathrm{~K}$, and $\eta_{0}^{\text {decay }}=1-3$, and setting $I / I_{0}=1 / \eta_{0}^{\text {decay }}$, we obtain $T=\Theta \simeq 326 \mathrm{~K}$, When $I / I_{0}>1 / \eta_{0}^{\text {decay }}$, we obtain $\varepsilon_{F} \simeq \varepsilon_{F}^{s a t} \simeq(7-17) \mathrm{meV}$, and $\exp \left(\varepsilon_{F}^{\text {sat }} / 2 T\right) \lesssim$ $1.07-1.19$.

The optical phonon decay time and, hence, parameter $\eta_{0}^{\text {decay }}$ might be small in GLs on properly chosen substrate. In such a case, the quasi-Fermi energy at sufficiently strong pumping can be not so small. Indeed, setting $I / I_{0}=1 / \eta_{0}^{\text {decay }}$ and $\eta_{0}^{\text {decay }}=0.1-0.5$, from Eqs. (15) - (17) we obtain $\varepsilon_{F}^{\text {sat }} \lesssim 17-45 \mathrm{meV}$, and $\exp \left(\varepsilon_{F}^{\text {sat }} / 2 T\right) \lesssim 1.19-1.57$.

The optical phonon decay time, particularly in suspended GLs, can be fairly long (in the range 1-10 ps 21$23,31[33]$ ), and it might markedly exceed the characteristic time of intraband interaction of electrons and holes with optical phonons. Hence, the inequality $\eta_{0}^{\text {decay }} \gg$ $\eta_{0}>1$ can, be valid, particularly, in the case of suspended GLs. In this fairly realistic case, Eqs. (12) - (14) lead to Eqs. (14) - (17) and their consequences at all values of $\Omega_{0} / \omega_{0}$, because the terms with $\eta_{0}\left(\Omega_{0} / \omega_{0}-1\right)$ are relatively small and can be omitted.

Thus, at long optical phonon decay times, the effect of accumulation of optical phonons (heating of the opti- 
cal phonon system) can prevent realization of population inversion necessary for lasing (large quasi-Fermi energy $\left.\varepsilon_{F}\right)$ at room temperatures when the processes involving optical phonons can dominate.

\section{B. Weak pumping}

At sufficiently weak pumping (see below), Eqs. (12) - (14) can be solved analytically at arbitrary values of $\eta_{0}^{\text {decay }}, \eta_{0}$, and $\Omega_{0} / \omega_{0}$. In this case, the effective temperature and quasi-Fermi energy are close to $T_{0}$ and zero, respectively. As shown in the Appendix, at $\varepsilon_{F}<T$, one obtains $\eta_{0}=\eta_{0}^{e q} \simeq\left(\hbar \omega_{0} / \pi T_{0}\right)^{2} /\left(1+2.19 T_{0} / \hbar \omega_{0}\right)$. At room temperature, $\eta_{0}^{e q} \simeq 5$. Thus, at a weak pumping,

$$
\begin{gathered}
T \simeq T_{0}+\frac{T_{0}^{2}}{\hbar \omega_{0}}\left[\eta_{0}^{\text {decay }}+\eta_{0}^{e q}\left(\frac{\Omega_{0}}{\omega_{0}}-1\right)\right] \frac{I}{I_{0}} \\
\simeq T_{0}+\frac{T_{0}^{2}}{\hbar \omega_{0}}\left[\eta_{0}^{\text {decay }}+5\left(\frac{\Omega_{0}}{\omega_{0}}-1\right)\right] \frac{I}{I_{0}} \\
\varepsilon_{F} \simeq \frac{T_{0}}{2}\left[1-\eta_{0}^{e q}\left(\frac{\Omega_{0}}{\omega_{0}}-1\right)\right] \frac{I}{I_{0}} \\
\simeq 3 T_{0}\left(1-\frac{5}{6} \frac{\Omega_{0}}{\omega_{0}}\right) \frac{I}{I_{0}} .
\end{gathered}
$$

Simultaneously, one obtains

$$
\begin{aligned}
\exp \left(\frac{2 \varepsilon_{F}}{T}\right) & \simeq 1+\left[1-\eta_{0}^{e q}\left(\frac{\Omega_{0}}{\omega_{0}}-1\right)\right] \frac{I}{I_{0}} \\
& \simeq 1+3\left(1-\frac{5}{6} \frac{\Omega_{0}}{\omega_{0}}\right) \frac{I}{I_{0}} .
\end{aligned}
$$

Equations (19) - (21) are valid if

$$
I \ll \frac{I_{0}}{\left|\eta_{0}^{\text {decay }}+\eta_{0}^{e q}\left(\frac{\Omega_{0}}{\omega_{0}}-1\right)\right|}=\frac{I_{0}}{\left|\eta^{e q}\right|},
$$

where $\eta^{e q}=\eta_{0}^{\text {decay }}+\eta_{0}^{e q}\left(\Omega_{0} / \omega_{0}-1\right)$.

As clearly seen from Eqs. (19) and (20) (as well as from Eqs. (13) and (14)), the effective temperature increases (the electron and hole heating) with increasing $I$ if $\eta^{e q}>0$, i.e., if $\Omega_{0} / \omega_{0}>1-\eta_{0}^{\text {decay }} / \eta_{0}^{e q}$. The rise of the effective temperature is more pronounced at larger $\eta_{0}^{\text {decay }}$, i.e., at longer decay time $\tau_{0}^{\text {decay }}$. Under the condition $\Omega_{0} / \omega_{0}>1+1 / \eta_{0}^{e q} \simeq 6 / 5$, the quasi-Fermi energy can become negative $\varepsilon_{F}<0$, so that $\exp \left(\varepsilon_{F} / T\right)<1$. In the latter case, the optical pumping does not lead to the degeneration of the electron-hole plasma. It is interesting that this conclusion is independent of the relative value of the decay time $\tau_{0}^{\text {decay }}$.
However, at sufficiently small ratio $\Omega_{0} / \omega_{0}$, parameter $\eta^{e q}$ can be negative, and the effective temperature can decrease (cooling of the electron-hole plasma). If $\Omega_{0} / \omega_{0}<6 / 5$, the quasi-Fermi energy is a rising function of the pumping intensity. The latter inequality implies that there should be $\Omega<\omega_{0}(2 K+1.2)$. The latter conditions of the electron-hole plasma cooling accompanied with the quasi-Fermi energy increase do not contradict inequalities Eq. (5). The effect of cooling under consideration is attributed to the effective photogeneration of low-energy electrons and holes (with the energy $\hbar \Omega_{0} / 2$ )

\section{Strong pumping, $\Omega_{0} / \omega_{0}>1-\eta_{0}^{e q} / \eta_{0}^{\text {decay }}$.}

In this case, $\eta_{0}^{\text {decay }}+\eta_{0}^{e q}\left(\Omega_{0} / \omega_{0}-1\right)>0$, even at elevated pumping intensities $I \gtrsim I_{0}$, a moderate increase in $T$ and $\left|\varepsilon_{F}\right|$ exhibit a moderate increase with increasing $I / I_{0}$, which slows down in the range of large $I / I_{0}$ (the $T-I$ dependence becomes logarithmic, whereas the quasi-Fermi energy tends to a constant value, positive or negative). Indeed, in the case under consideration, Eq. (14) does not have solutions with $\varepsilon_{F} \gg T$. Hence in the logarithm in Eq. (14) one can put $\eta_{0}=\eta_{0}^{e q} \simeq 5$. As a result, we obtain

$$
\begin{gathered}
T \simeq T_{0}+\left(\frac{T_{0}^{2}}{\hbar \omega_{0}}\right) \ln \left\{\left[\eta_{0}^{\text {decay }}+\eta_{0}^{e q}\left(\frac{\Omega_{0}}{\omega_{0}}-1\right)\right] \frac{I}{I_{0}}\right\} \\
\simeq T_{0}+\left(\frac{T_{0}^{2}}{\hbar \omega_{0}}\right) \ln \left\{\left[\eta_{0}^{\text {decay }}+5\left(\frac{\Omega_{0}}{\omega_{0}}-1\right)\right] \frac{I}{I_{0}}\right\}, \quad \\
\varepsilon_{F} \simeq \frac{T_{0}}{2} \ln \left[\frac{\eta_{0}^{\text {decay }}+1}{\eta_{0}^{\text {decay }}+\eta_{0}^{e q}\left(\frac{\Omega_{0}}{\omega_{0}}-1\right)}\right] \\
\simeq \frac{T_{0}}{2} \ln \left[\frac{\eta_{0}^{\text {decay }}+1}{\eta_{0}^{\text {decay }}+5\left(\frac{\Omega_{0}}{\omega_{0}}-1\right)}\right]=\varepsilon_{F}^{\text {sat }} .
\end{gathered}
$$

When $\eta_{0}^{\text {decay }} \gg 1$, Eq. (23) yields

$$
\begin{aligned}
\varepsilon_{F}^{s a t} \simeq & \frac{T_{0}}{2} \ln \left[1+\frac{5}{\eta_{0}^{\text {decay }}}\left(\frac{6}{5}-\frac{\Omega_{0}}{\omega_{0}}\right)\right] \\
& \simeq T_{0} \frac{5}{2 \eta_{0}^{\text {decay }}}\left(\frac{6}{5}-\frac{\Omega_{0}}{\omega_{0}}\right) .
\end{aligned}
$$

As follows from Eqs. (20), (23), and (24), $\varepsilon_{F}$ changes its sign at $\Omega_{0} / \omega_{0}=6 / 5$ both at weak and relatively strong pumping. In the case under consideration, the population inversion is weak ( $\varepsilon_{F}$ can be positive, but small) or is not realized $\left(\varepsilon_{F}<0\right)$. 
Formally, the logarithmic factors in Eqs. (22) and (23) diverge when $\Omega_{0} / \omega_{0}$ tends to $1-\eta_{0}^{e q} / \eta_{0}^{\text {decay }}$. However, these equations become invalid in the immediate vicinity of the point $\Omega_{0} / \omega_{0}=1-\eta_{0}^{e q} / \eta_{0}^{\text {decay }}$, because above we have assumed $\varepsilon_{F} / T<1$ and put $\eta_{0}=\eta_{0}^{e q}$. The case of smaller $\Omega_{0} / \omega_{0}$ is considered in the next subsection. at sufficiently large $I / I_{0}$. In such an instance, one can expect that $\varepsilon_{F} \gg T$ and $\eta_{0} \simeq\left(\hbar \omega_{0} / \varepsilon_{F}\right) / 6$. Hence, Eq. (14) can be presented as

\section{Strong pumping, small $\Omega_{0} / \omega_{0}$ (cooling regime).}

The case $\Omega_{0} / \omega_{0}$ is small, is much more interesting because rather large values of $\varepsilon_{F}$ and $\varepsilon_{F} / T$ can be achieved

$$
\begin{aligned}
& T \simeq \frac{T_{0}}{1-\frac{T_{0}}{\hbar \omega_{0}} \ln \left\{1+\left[\eta_{0}^{\text {decay }}+\frac{1}{6}\left(\frac{\hbar \omega_{0}}{\varepsilon_{F}}\right)^{2}\left(\frac{\Omega_{0}}{\omega_{0}}-1\right)\right] \frac{I}{I_{0}}\right\}} \\
& \varepsilon_{F} \simeq \frac{T_{0}}{2} \frac{\ln \left\{\frac{1+\left(\eta_{0}^{\text {decay }}+1\right) \frac{I}{I_{0}}}{\left.1+\left[\eta_{0}^{\text {decay }}+\frac{1}{6}\left(\frac{\hbar \omega_{0}}{\varepsilon_{F}}\right)^{2}\left(\frac{\Omega_{0}}{\omega_{0}}-1\right)\right] \frac{I}{I_{0}}\right\}}\right.}{1-\frac{T_{0}}{\hbar \omega_{0}} \ln \left\{1+\left[\eta_{0}^{\text {decay }}+\frac{1}{6}\left(\frac{\hbar \omega_{0}}{\varepsilon_{F}}\right)^{2}\left(\frac{\Omega_{0}}{\omega_{0}}-1\right)\right] \frac{I}{I_{0}}\right\}} .
\end{aligned}
$$

Assuming that $\varepsilon_{F} \gg T$, one can approximately solve Eqs. (25) and (26) by iterations, considering the smallness of $\exp \left(-\varepsilon_{F} / T\right)$, and arrive at

$$
\begin{gathered}
T \simeq T_{0}\left[1-2 \sqrt{\left.\frac{\left(1-\frac{\Omega_{0}}{\omega_{0}}\right) \frac{I}{I_{0}}}{6\left(1+\eta_{0}^{\text {decay }} \frac{I}{I_{0}}\right)}\right]},\right. \\
\varepsilon_{F} \simeq \hbar \omega_{0} \sqrt{\frac{\left(1-\frac{\Omega_{0}}{\omega_{0}}\right) \frac{I}{I_{0}}}{6\left(1+\eta_{0}^{\text {decay }} \frac{I}{I_{0}}\right)}} .
\end{gathered}
$$

If $I / I_{0} \gg 1 / \eta_{0}^{\text {decay }}$, from Eqs. (27) and (28) for $T$ and $\varepsilon_{F}$ we obtain their saturation values: $T^{\text {sat }} \simeq T_{0}\left[1-2 \sqrt{\left(1-\Omega_{0} / \omega_{0}\right) / 6 \eta_{0}^{\text {decay }}}\right]$ and $\varepsilon_{F}^{s a t} \simeq$ $\hbar \omega_{0} \sqrt{\left(1-\Omega_{0} / \omega_{0}\right) / 6 \eta_{0}^{\text {decay }}}$. The corrections to Eqs. $(27)$ and $(28)$ are exponentially small when $\Omega_{0} / \omega_{0}$ is not too close to unity. Due to the above assumptions, Eqs. (27) and (28) are valid until $2 \varepsilon_{F}<\hbar \omega_{0}$, that yields

$$
\left(\frac{2 T_{0}}{3 \hbar \omega_{0}}\right)^{2} \ll \frac{2\left(1-\Omega_{0} / \omega_{0}\right) I / I_{0}}{3\left(1+\eta_{0}^{\text {decay }}\right) I / I_{0}}<1 .
$$

Considering that $\left(2 T_{0} / 3 \hbar \omega_{0}\right)^{2} \simeq 4 / 576 \ll 1$, these equations are valid in rather wide range of pumping intensities, (not too small), except the case when $\left(1-\Omega_{0} / \omega_{0}\right)$ is very close to zero (this is the special case considered above). Some complications can arise in a non-realistic case of very small $\eta_{0}^{\text {decay }}$. Using Eqs. (27) and (28), one can find the condition when $\varepsilon_{F} \gg T$ that was assumed in deriving Eqs. (25) and (26) and their consequences (see also Eq. (5)):

$$
\frac{\Omega_{0}}{\omega_{0}}<1-6 \eta_{0}^{\text {decay }}\left(\frac{T_{0}}{\hbar \omega_{0}}\right)^{2} .
$$

At room temperature, the latter inequality yields $\Omega_{0} / \omega_{0}<1-0.1 \eta_{0}^{\text {decay }}$. This implies that large $\varepsilon_{F}$ and $T<T_{0}$ can be achieved if $\eta_{0}^{\text {decay }}<10$ and the ratio $\Omega_{0} / \omega_{0}$ is chosen properly.

As it was mentioned above, the effect of cooling under consideration is attributed to the effective photogeneration of low-energy electrons and holes.

Setting $T_{0}=300 \mathrm{~K}, \hbar \Omega=900 \mathrm{meV}$, we obtain $\hbar \Omega_{0} \simeq 0.1 \mathrm{eV}$ and $\Omega_{0} / \omega_{0} \simeq 0.5$. For this case, assuming $\eta_{0}^{\text {decay }}=1-3$, we obtain $T^{\text {sat }} \simeq 127-200 \mathrm{~K}$ and $\varepsilon_{F}^{\text {sat }} \simeq(33-58) \mathrm{meV}$. Hence, $\exp \left(\varepsilon_{F}^{\text {sat }} / 2 T\right) \simeq 2.7-15.5$. These latter two values are markedly larger than those obtained in Subsection A for $\Omega_{0} / \omega_{0}=1$. If $\eta_{0}^{\text {decay }}<1$, the effect of cooling is more pronounced and the quan- 


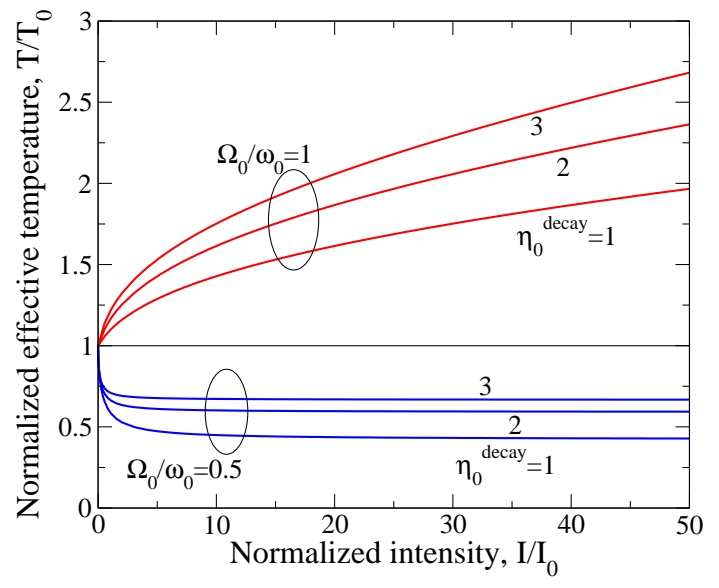

FIG. 1: Normalized carrier effective temperature $T / T_{0}$ versus normalized pumping intensity $I / I_{0}$ for different $\eta_{0}^{\text {decay }}$ and $\Omega_{0} / \omega_{0}$.

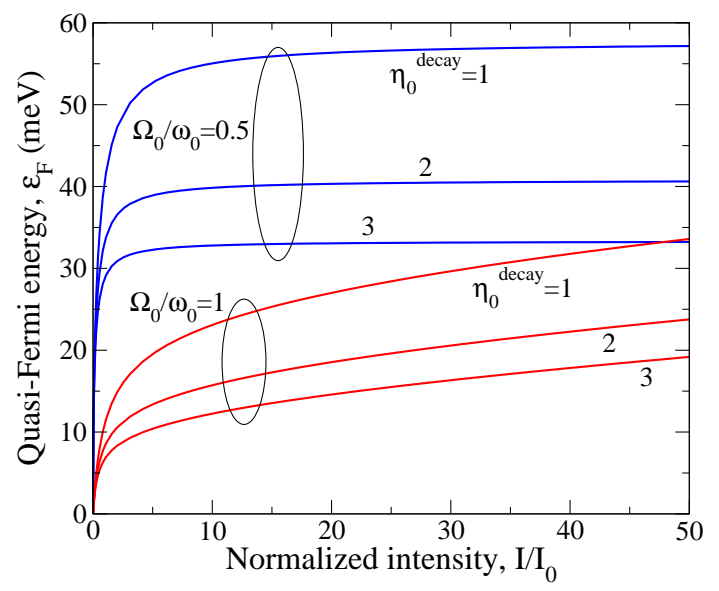

FIG. 2: Quasi-Fermi energy $\varepsilon_{F}$ versus normalized pumping intensity $I / I_{0}$ for different $\eta_{0}^{\text {decay }}$ and $\Omega_{0} / \omega_{0}$.

tities $\varepsilon_{F}^{s a t}$ and $\exp \left(\varepsilon_{F}^{s a t} / 2 T\right)$ can be fairly large. At the parameters chosen for the above estimates, conditions (5) are satisfied.

It is instructive that in the cooling regime in question, the optical phonon system is heated. The effective optical phonon temperature $\Theta$ in this regime is given by the same equation as in other regimes under consideration, i.e. by Eq. (18), which yields $\Theta \geq T_{0}$.

\section{CALCULATION OF THE GL DYNAMIC CONDUCTIVITY}

The attenuation and amplification of electromagnetic waves and surface plasmons propagating along the GL plane is determined by the the real part of the GL dy- namic ac conductivity at the signal frequency $\omega$ (photon or plasmon frequency) Re $\sigma_{\omega}$. The latter includes the contributions of the interband and intraband transitions:

$$
\operatorname{Re} \sigma_{\omega}=\operatorname{Re} \sigma_{\omega}^{i n t e r}+\operatorname{Re} \sigma_{\omega}^{i n t r a} .
$$

For the multiple-GL structures (see, for instance, Refs. 9, 34]), the contributions of all GL to $\operatorname{Re} \sigma_{\omega}$ should be summarized [11, 12].

In the situation under consideration, generalizing the pertinent formula from Ref. [6] (see, for instance, Refs. 2, 11]) for the photogenerated nonequilibrium electron hole plasma with the equal non-zero quasi-Fermi energies of electrons and holes, one can obtain:

$$
\operatorname{Re} \sigma_{\omega}^{\text {inter }}=\left(\frac{e^{2}}{4 \hbar}\right) \tanh \left(\frac{\hbar \omega-2 \varepsilon_{F}}{4 T}\right) .
$$

Here, $e=|e|$ is the electron charge. At $\omega \gg \tau^{-1}$, where $\tau$ is the momentum relaxation time, The intraband contribution, which actually corresponds to the Drude absorption by electrons and holes, can be obtained from the Boltzmann equation [2, 11, 35]:

$$
\operatorname{Re} \sigma_{\omega}^{\text {intra }}=\frac{2 e^{2}}{\pi \hbar^{2} \omega^{2}} \int_{0}^{\infty} \frac{d \varepsilon \varepsilon}{\tau} \frac{d}{d \varepsilon}\left\{-\frac{1}{\exp \left[\left(\varepsilon-\varepsilon_{F}\right) / T\right]+1}\right\} .
$$

As $T_{0}$ and $T$ are small in comparison with $\hbar \omega_{0}$, the electron and hole momentum relaxation is associated with different scattering mechanisms, in particular, with the scattering on short-range and long-range disorder and acoustic phonons. In this case, $\tau^{-1}=\nu_{0}\left(\varepsilon / T_{0}\right),\left(\nu_{0}\right.$ is the collision frequency of electron and holes with the thermal equilibrium energy), so that interpolating the integral in Eq. (31) [see Eq. (A10)] as a function of $\varepsilon$ and $T$, from Eq. (31) we obtain

$$
\operatorname{Re} \sigma_{\omega}^{i n t r a} \simeq \frac{2 e^{2} \nu_{0}}{\pi \hbar^{2} \omega^{2}} \frac{\left(\varepsilon_{F}^{2}+\pi^{2} T^{2} / 6\right)}{T_{0}} .
$$

When $\varepsilon_{F} \gg T$ from Eqs. (31) and (32) we obtain

$$
\operatorname{Re} \sigma_{\omega}^{i n t r a} \simeq \frac{2 e^{2} \nu_{0}}{\pi \hbar^{2} \omega^{2}} \frac{\varepsilon_{F}^{2}}{T_{0}}
$$

Hence, using Eqs. (30) - (33), we arrive at

$\operatorname{Re} \sigma_{\omega}=\left(\frac{e^{2}}{4 \hbar}\right)\left[\tanh \left(\frac{\hbar \omega-2 \varepsilon_{F}}{4 T}\right)+\frac{8 \nu_{0}}{\pi \hbar \omega^{2}} \frac{\left(\varepsilon_{F}^{2}+\pi^{2} T^{2} / 6\right)}{T_{0}}\right]$.

One can see that at $\tau^{-1} \propto \varepsilon$, the Drude term given by Eqs. (34) and (35) is proportional to the density of the electron-hole plasma.

In the special case considered above when $\Omega_{0} / \omega_{0}=1$, using Eqs. (16), (17), and (34), we obtain 


$$
\begin{gathered}
\frac{\operatorname{Re} \sigma_{\omega}}{\sigma_{0}}=\frac{\frac{\exp \left(\hbar \omega / 2 T_{0}\right)}{\frac{\left(1+\eta_{0}^{\text {decay }} I / I_{0}\right) \omega / 2 \omega_{0}}{\exp \left(\hbar \omega / 2 T_{0}\right)}-\left(1+\frac{I / I_{0}}{1+\eta_{0}^{\text {decay }} I / I_{0}}\right)^{1 / 2}}+\left(1+\frac{I / I_{0}}{1+\eta_{0}^{\text {decay }} I / I_{0}}\right)^{1 / 2}}{\left(1+\eta_{0}^{\text {decay }} I / I_{0}\right)^{\omega / 2 \omega_{0}}} \\
+\left(\frac{\omega_{D}}{\omega}\right)^{2}\left\{\frac{3}{2 \pi^{2}} \ln ^{2}\left(1+\frac{I / I_{0}}{1+\eta_{0}^{\text {decay }} I / I_{0}}\right)+\left[1-\frac{T_{0}}{\hbar \omega_{0}} \ln \left(1+\eta_{0}^{\text {decay }} I / I_{0}\right)\right]^{-2}\right\} .
\end{gathered}
$$

Here $\sigma_{0}=e^{2} / 4 \hbar$ and $\omega_{D}=\sqrt{4 \pi \nu_{0} T_{0} / 3 \hbar}$. In the THz and middle IR ranges, $\omega \ll 2 \omega_{0}\left(\omega / 2 \pi \ll 10^{3} \mathrm{THz}\right)$, Eq. (35) can be reduced to the following:

$$
\begin{gathered}
\frac{\operatorname{Re} \sigma_{\omega}}{\sigma_{0}}=\frac{\exp \left(\frac{\hbar \omega}{2 T_{0}}\right)-\left(1+\frac{I / I_{0}}{1+\eta_{0}^{\text {decay }} I / I_{0}}\right)^{1 / 2}}{\exp \left(\frac{\hbar \omega}{2 T_{0}}\right)+\left(1+\frac{I / I_{0}}{1+\eta_{0}^{\text {decay }} I / I_{0}}\right)^{1 / 2}} \\
+\left(\frac{\omega_{D}}{\omega}\right)^{2}\left\{\frac{3}{2 \pi^{2}} \ln ^{2}\left(1+\frac{I / I_{0}}{1+\eta_{0}^{\text {decay }} I / I_{0}}\right)+\left[1-\frac{T_{0}}{\hbar \omega_{0}} \ln \left(1+\eta_{0}^{\text {decay }} I / I_{0}\right)\right]^{-2}\right\} .
\end{gathered}
$$

Assuming $\nu_{0}=(0.25-1.0) \times 10^{12} \mathrm{~s}^{-1}$ at $T_{0}=300 \mathrm{~K}$, we obtain $f_{D}=\omega_{D} / 2 \pi \simeq(1-2) \mathrm{THz}$. Considering that the momentum relaxation time in multiple-GLs at the temperatures $T_{0} \leq 50 \mathrm{~K}$ can reach values about $20 \mathrm{ps}$ [34], the above values of $\nu_{0}$ at $T_{0}=300 \mathrm{~K}$ appears to be reasonable (assuming a linear increase of $\nu_{0}$ with the lattice temperature $T_{0}[35]$ ).

When $\Omega_{0} / \omega_{0}$ is small, using Eqs. (27), (28), and (34), we arrive at

$$
\begin{array}{r}
\frac{\operatorname{Re} \sigma_{\omega}}{\sigma_{0}}=\frac{\exp \left[\frac{\hbar \omega}{2 T_{0}} \frac{1}{(1-2 Z)}\right]-\exp \left[\frac{\hbar \omega_{0}}{T_{0}} \frac{Z}{(1-2 Z)}\right]}{\exp \left[\frac{\hbar \omega}{2 T_{0}} \frac{1}{(1-2 Z)}\right]+\exp \left[\frac{\hbar \omega_{0}}{T_{0}} \frac{Z}{(1-2 Z)}\right]} \\
+\left(\frac{\omega_{D}}{\omega}\right)^{2}\left[\frac{6}{\pi^{2}}\left(\frac{\hbar \omega_{0}}{T_{0}}\right)^{2} Z^{2}+(1-2 Z)^{2}\right] .
\end{array}
$$

Here $Z=\sqrt{\left[\left(1-\Omega_{0} / \omega_{0}\right) I / I_{0}\right] /\left(1+\eta_{0}^{\text {decay }} I / I_{0}\right)}$.

\section{DISCUSSION OF THE RESULTS.}

As follows from Sec. V, to achieve the population inversion sufficiently strong for lasing, the parameter $\Omega_{0} / \omega_{0}$ should be either equal to unity (the special case) or smaller than unity (cooling regime). Below we shall focus on this situation.
Figure 1 shows the dependences of the normalized effective temperature $T / T_{0}$ on the normalized pumping intensity $I / I_{0}$ calculated for different $\eta_{0}^{\text {decay }}$ using eq. (15) for $\Omega_{0} / \omega_{0}=1$ and Eq. (27) for $\Omega_{0} / \omega_{0}=0.5$. Figure 2 shows the dependences of the quasi-Fermi energy $\varepsilon_{F}$ on the normalized pumping intensity $I / I_{0}$ calculated for different $\eta_{0}^{\text {decay }}$ and $\Omega_{0} / \omega_{0}$ using Eqs. (16) and (28).

One can see from Figs. 1 and 2 that at $\Omega_{0} / \omega_{0}<1$, a pronounced cooling of the electron-hole plasma takes place, and this cooling regime provides larger $\varepsilon_{F}$ for a given pumping intensity. As also follows from Figs 1 and 2, the finiteness of the optical decay time markedly affects the $T-I$ and $\varepsilon_{F}-I$ dependences. Indeed, at $\Omega_{0} / \omega_{0}=1$, the $T-I$ dependence becomes steeper when the parameter $\eta_{0}^{\text {decay }}$ increases (reinforcement of heating effect), whereas at $\Omega_{0} / \omega_{0}<1$, this dependence becomes less pronounced (weakening of cooling). This is attributed to the optical phonon heating, which promotes the heating of the electron-hole plasma at $\Omega_{0} / \omega_{0}=1$ and counteract its cooling at $\Omega_{0} / \omega_{0}<1$.

Figure 3 demonstrates the dependences of the real part of the GL dynamic conductivity $\operatorname{Re} \sigma_{\omega}$ on the signal frequency $\omega / 2 \pi$ at different normalized pumping intensities $I / I_{0}$ calculated for different $\eta_{0}^{\text {decay }}$ and $\nu_{0}$ using Eq. (35) (assuming that $\Omega_{0} / \omega_{0}=1$ ). As seen from Fig. 3, at $\Omega_{0} / \omega_{0}=1$, the dynamic conductivity $\operatorname{Re} \sigma_{\omega}$ can be negative only if parameter $\eta_{0}^{\text {decay }} \ll 1$ (the optical phonon decay time is small), i.e., if the heating of electrons, holes, and optical phonons is very weak. However, at $\Omega_{0} / \omega_{0}<1$ when electron-hole plasma is cooled, $\operatorname{Re} \sigma_{\omega}<0$ in range 


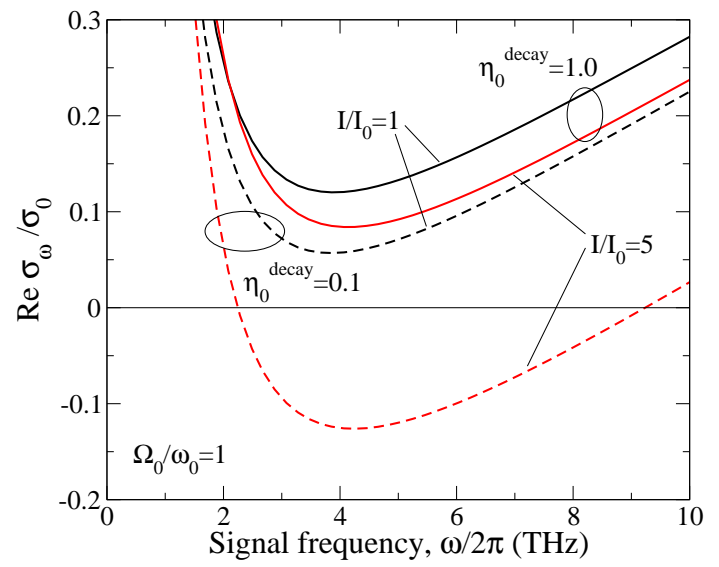

FIG. 3: Real part of the normalized dynamic conductivity as a function of signal frequency at different normalized pumping intensities $I / I_{0}$ and different $\eta_{0}^{\text {decay }}\left(\Omega_{0} / \omega_{0}=1\right.$ and $\nu_{0}=$ $\left.0.25 \times 10^{12} \mathrm{~s}^{-1}\right)$.

of signal frequencies from $\omega / 2 \pi \simeq 2 \mathrm{THz}$ to $17 \mathrm{THz}$ and more [see Figs. 4 and 5, obtained using Eq. (37)]. This occurs at not too low pumping intensities $\left(I / I_{0}=5\right)$ but even at rather large values of $\nu_{0}$ and $\eta_{0}^{\text {decay }}$ when the cooling of the electron-hole plasma is accompanied by the heating of the optical phonon system.

Figures 6 and 7 calculated using Eq. (37) show the real part of the GL dynamic conductivity Re $\sigma_{\omega}$ versus the normalized pumping intensity $I / I_{0}$ calculated for given values of the signal frequency $\omega / 2 \pi=2 \mathrm{THz}$ (at $\nu_{0}=2.5 \times 10^{11} \mathrm{~s}^{-1}$ ) and $\omega / 2 \pi=3.5 \mathrm{THz}$. (at $\left.\nu_{0}=1 \times 10^{12} \mathrm{~s}^{-1}\right)$. In both cases, $\operatorname{Re} \sigma_{\omega}$ is negative when $I / I_{0}$ exceeds certain threshold value. The latter is somewhat smaller in GL with smaller $\nu_{0}$. It is interesting that in the cases $\nu_{0}^{\text {decay }}=1$, an increase in $I / I_{0}$ leads to smaller $\left|\operatorname{Re} \sigma_{\omega}\right|$ in the range of large $I / I_{0}$, in contrast to the cases $\nu_{0}^{\text {decay }}=2$ and $\nu_{0}^{\text {decay }}=3$, This can be explained by a faster increase in $\varepsilon_{F}$ with increasing $I / I_{0}$ at smaller $\nu_{0}^{\text {decay }}$ leading to a faster increase in the Drude absorption.

To estimate $I_{0}$, we use the data for $\Sigma_{0} / \tau_{0}^{\text {inter }}$ at $T_{0}=300 \mathrm{~K}$ from Ref. 15] (see Appendix) and set $\hbar \Omega_{0}=100 \mathrm{meV}$, so that $\left(\Omega_{0} / \omega_{0} \simeq 0.5\right)$. and setting $\hbar \Omega_{0}=100 \mathrm{meV}$. The chosen value of $\hbar \Omega_{0}$ can correspond to the optical pumping photon energy $\hbar \Omega=900 \mathrm{meV}$ provided the cascade emission of $K=2$ optical phonons or to $\hbar \Omega=\hbar \Omega_{0}=100 \mathrm{meV}$ (pumping by $\mathrm{CO}_{2}$ laser). In such cases, one obtains $I_{0} \simeq 9,66 \times 10^{21} 1 / \mathrm{cm}^{2} \mathrm{~s}$ and $I_{0} \simeq 8.69 \times 10^{22} 1 / \mathrm{cm}^{2} \mathrm{~s}$, respectively. In both cases, $S_{0}=\hbar \Omega I_{0} \simeq 1.39 \times 10^{4} \mathrm{~W} / \mathrm{cm}^{2}$.

As demonstrated, lowering of the ration $\Omega_{0} / \omega_{0}$ is beneficial for achieving the population inversion and negative dynamic conductivity, because such lowering results in weakening of the electron-hole plasma heating and even in the transition to its cooling. Moreover, as follows from Eq. (23) and (24), at elevated $\Omega_{0} / \omega_{0}$, the value of the

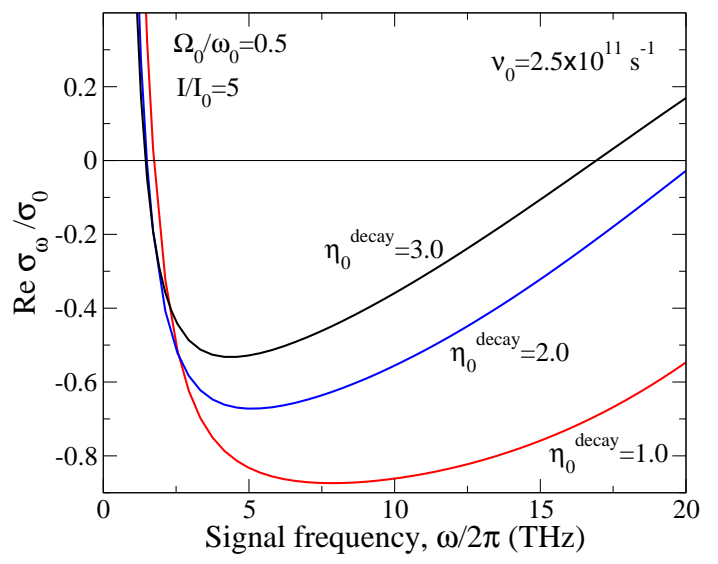

FIG. 4: Real part of the normalized dynamic conductivity as a function of signal frequency at different normalized pumping intensities $I / I_{0}$ and different $\eta_{0}^{\text {decay }}\left(\Omega_{0} / \omega_{0}=0.5\right.$ and $\nu_{0}=$ $\left.2.5 \times 10^{11} \mathrm{~s}^{-1}\right)$.

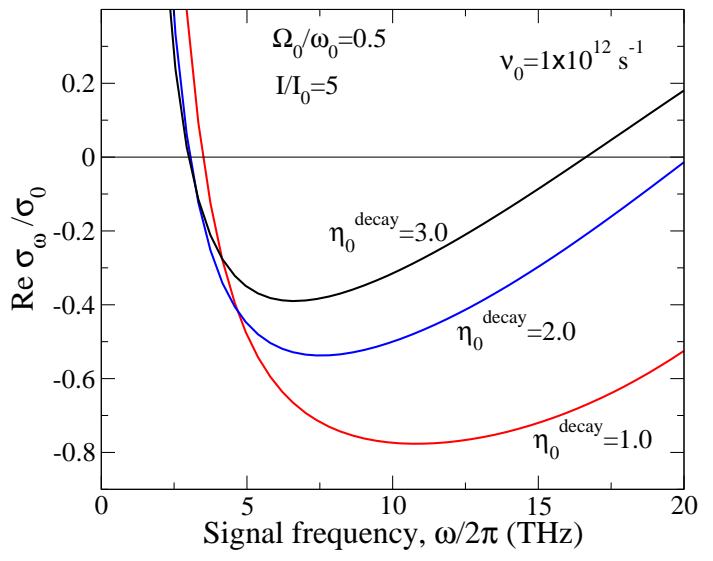

FIG. 5: The same as in Fig. 4 but for $\nu_{0}=1.0 \times 10^{12} \mathrm{~s}^{-1}$.

quasi-Fermi energy $\varepsilon_{F}$ can become negative that corresponds to nondegenerate electron-hole plasma $(f<0.5)$. In this regard, in the case of optical pumping with relatively high photon energy, the suppression of the pair carrier interactions might be indispensable for the realization of strong population inversion and lasing. This, possibly, can be done using the GL structures with high-k substrates.

Instead of the optical pumping under consideration, the electron-hole cooling and the realization of strong population inversion can be achieved by the injection pumping (in GL structures with p-n or p-i-n junctions) considered previously [3]. In such a case, one can put $\Omega_{0}=e V / \hbar \ll \omega_{0}$, if the applied bias voltage $V$ is not large $(V \ll 200 \mathrm{mV})$.

Above we assumed for simplicity that there is only one type of optical phonons with $\hbar \omega_{0} \simeq 200 \mathrm{meV}$. Actually, the processes involving the optical phonons with $\omega_{1}=$ $\left.\omega_{0}\right)$ and $\omega_{2}=\omega_{0}-\Delta \omega_{0}$ are important [16] $\left(\Delta \omega_{0} / \omega_{0} \simeq\right.$ 


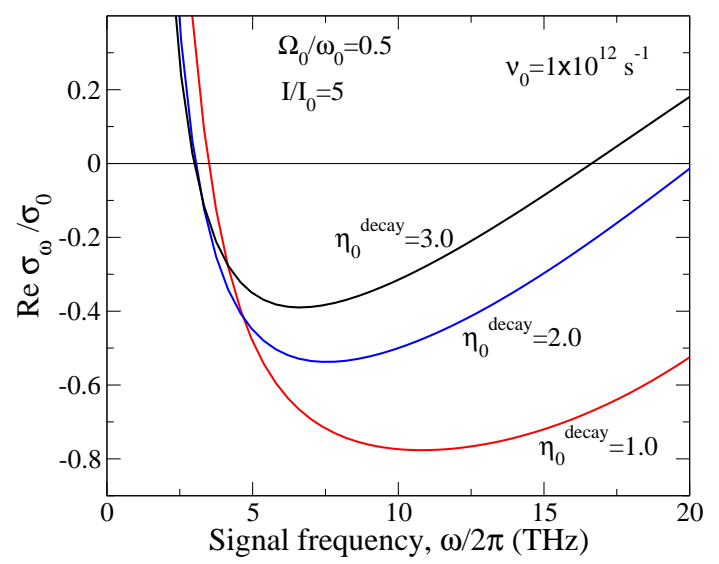

FIG. 6: Real part of the normalized dynamic conductivity as a function of normalized pumping intensities $I / I_{0}$ for $\omega / 2 \pi=$ $2 \mathrm{THz}$ and different $\eta_{0}^{\text {decay }}\left(\Omega_{0} / \omega_{0}=0.5\right.$ and $\nu_{0}=2.5 \times$ $\left.10^{11} \mathrm{~s}^{-1}\right)$.

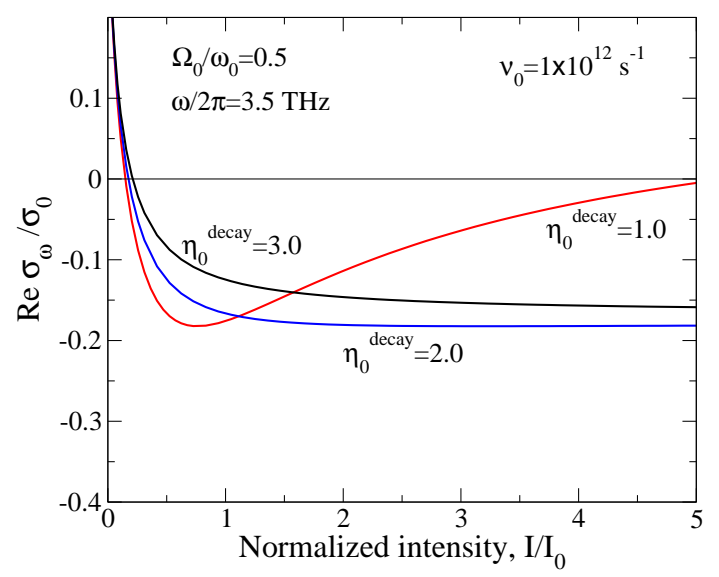

FIG. 7: The same as in Fig. 6 but for for $\omega / 2 \pi=3.5 \mathrm{THz}$ and $\nu_{0}=1.0 \times 10^{12} \mathrm{~s}^{-1}$.

0.18). This adds complexity to the pattern of the electron and hole relaxation affecting the value of parameter $\Omega_{0}$ and

Although one can expect that due to a smallness of $\Delta \omega_{0} / \omega_{0}$, this effect is not essential and can be accounted for by a proper correction of $\Omega_{0}$. . More complicating factor can be the interaction of electrons and holes with optical phonons in the substrate. These phonons, having relatively low energy, can, in principle, markedly contribute to the recombination and energy relaxation. Perhaps in multiple GL-structures the effect substrate phonons is not pronounced. The pertinent generalization of our treatment is going to be considered elsewhere.

Lowering of the lattice temperature $T_{0}$ and, consequently, the effective temperature $T$ results a weakening of the efficiency of optical phonon assisted processes, particularly, in a decrease in the recombination rate [see Eqs. (6) and (7)]. Apart from this, the Drude absorption also becomes smaller due to a decrease in $\nu_{0}$ [see
Eq. (34)]. Therefore, although, as demonstrated above, the $\mathrm{THz}$ and IR lasing using the population inversion in graphene can be realized even at room temperatures, at lower temperatures, it can be achieved easier. However, at lower temperatures, the optical phonon assisted processes in GLs can give way to other recombination, generation, and energy relaxation mechanisms, for instance, the radiative recombination and generation and the energy relaxation on acoustic phonons [13, 14, 36]. To estimate the temperature range, in which the above simplified "optical phonon" model is sufficient, we assume that the characteristic time of radiative recombination and that associated with the optical phonon emission are about $(10-20) \mathrm{ns}$ and $0.5 \mathrm{ps}$, respectively. In this case, one can obtain $T_{0}>220-230 \mathrm{~K}$, so that our model is valid at room temperatures and slightly lower.

\section{CONCLUSIONS}

We have considered the characteristics of electron-hole plasma in optically pumped GL at elevated (room) temperatures (its quasi-Fermi energy, effective temperature, and dynamic conductivity) and shown that:

(1) The interband and intraband processes of emission and absorption of optical phonons play a crucial role in the characteristics of optically pumped electron-hole plasma in graphene at elevated temperatures;

(2) The electron-hole plasma in optically pumped graphene can be both heated and cooled depending on parameter $\Omega_{0} / \omega_{0}$, while the optical phonon system is always heated;

(3) The accumulation of nonequilibrium optical phonons (their heating) due to finiteness of their decay time leads to a slower dependence of the electron and hole quasiFermi energy on the optical pumping intensity;

(4) The effects in question can markedly influence the achievement of the negative dynamic conductivity in optically pumped GLs associated with the population inversion and, hence, the realization of $\mathrm{THz}$ or IR lasing in the GL structures at room temperatures. The latter requires a careful choice of parameter $\Omega_{0} / \omega_{0}$ and minimization of parameter $\eta_{0}^{\text {decay }}$.

\section{ACKNOWLEDGMENT}

The work was supported by the Japan Science and Technology Agency, CREST, Japan.

\section{Appendix}

For the term $R_{0}^{\text {inter }}$ in Eq. (6), which describes the electron-hole recombination and generation processes assisted by optical phonons, one can use the following simplified formula: 


$$
\begin{gathered}
R^{\text {inter }}=\frac{G_{0}}{T_{0}^{3}} \int_{0}^{\hbar \omega_{0}} \frac{d \varepsilon \varepsilon\left(\hbar \omega_{0}-\varepsilon\right)}{\left[1+\exp \left(\frac{\varepsilon-\varepsilon_{F}}{T}\right)\right]\left[1+\exp \left(\frac{\hbar \omega_{0}-\varepsilon-\varepsilon_{F}}{T}\right)\right]}\left[\mathcal{N}_{0}+1-\exp \left(\frac{\hbar \omega_{0}-2 \varepsilon_{F}}{T}\right) \mathcal{N}_{0}\right] \\
=\frac{G_{0}\left(\hbar \omega_{0}\right)^{2} T}{6 T_{0}^{3}}\left[\left(\mathcal{N}_{0}+1\right) \exp \left(\frac{2 \varepsilon_{F}-\hbar \omega_{0}}{T}\right)-\mathcal{N}_{0}\right] \int_{\exp \left(-\varepsilon_{F} / T\right)}^{\exp \left[\left(\hbar \omega_{0}-\varepsilon_{F}\right) / T\right]} \frac{d u}{(1+u)\left[1+u e^{\left(\varepsilon_{F}-\hbar \omega_{0}\right) / T}\right]} \\
=\frac{G_{0}\left(\hbar \omega_{0}\right)^{2} T}{6 T_{0}^{3}}\left[\left(\mathcal{N}_{0}+1\right) \exp \left(\frac{2 \varepsilon_{F}-\hbar \omega_{0}}{T}\right)-\mathcal{N}_{0}\right] \frac{\exp \left(\frac{\hbar \omega_{0}-2 \varepsilon_{F}}{T}\right)}{\left[\exp \left(\frac{\hbar \omega_{0}-2 \varepsilon_{F}}{T}\right)-1\right]} \ln \left\{\exp \left(-\frac{\hbar \omega_{0}}{T}\right)\left[\frac{\exp \left(\frac{\hbar \omega_{0}-\varepsilon_{F}}{T}\right)+1}{\exp \left(-\frac{\varepsilon_{F}}{T}\right)+1}\right]^{2}\right\} .
\end{gathered}
$$

Equation (A1) can be markedly simplified in the most interesting situations when $\hbar \omega_{0}>2 \varepsilon_{F}, T$ :

$$
R^{\text {inter }} \simeq \frac{\Sigma_{0}}{\tau_{0}^{\text {inter }}}\left[\left(\mathcal{N}_{0}+1\right) \exp \left(\frac{2 \varepsilon_{F}-\hbar \omega_{0}}{T}\right)-\mathcal{N}_{0}\right]
$$

with

$$
\frac{\Sigma_{0}}{\tau_{0}^{\text {inter }}}=\frac{G_{0}\left(\hbar \omega_{0}\right)^{3}}{6 T_{0}^{3}} .
$$

Here we have neglected the terms in the preexponential factor with the ration $\varepsilon_{F} / \hbar \omega_{0}$ and introduced the rate of the thermal generation of the electron-hole pairs due to absorption of optical phonons in equilibrium $\left.G_{0}^{e q}=G_{0}\left(\hbar \omega_{0}\right)^{3} / 6 T_{0}^{3} \exp \left(-\hbar \omega_{0}\right) / T\right)=$ $\left(\Sigma_{0} / \tau_{0}^{\text {inter }}\right) \exp \left(-\hbar \omega_{0} / T\right)$. The latter was estimated in Ref. [16]: $G_{0}^{e q} \simeq 10^{21} \mathrm{~cm}^{-2} \mathrm{~s}^{-1}$ at $T_{0}=300 \mathrm{~K}$.

Equation (A2) differs from those in Refs. [11, 16] by the inclusion of the terms with $\mathcal{N}_{0}$, which correspond to the processes of stimulated emission and absorption of optical phonons At temperatures, $T \ll \hbar \omega_{0} \simeq 2300 \mathrm{~K}$, when the number of equilibrium optical phonons $\mathcal{N}_{0}=$ $\mathcal{N}_{0}^{e q} \ll 1$ (stimulated emission and absorption of optical phonons is negligible), eq. (A2) reduces to that obtained in ref. [11].

The term $R_{0}^{\text {intra }}$ in Eq. (7) can be calculated as

$$
R_{0}^{\text {intra }}=\frac{2 G_{0}}{T_{0}^{3}} \int_{0}^{\infty} \frac{d \varepsilon \varepsilon\left(\varepsilon+\hbar \omega_{0}\right)\left[\mathcal{N}_{0}+1 \exp \left(-\frac{\hbar \omega_{0}}{T}\right)-\mathcal{N}_{0}\right]}{\left[1+\exp \left(\frac{\varepsilon-\varepsilon_{F}}{T}\right)\right]\left[1+\exp \left(-\frac{\varepsilon-\varepsilon_{F}+\hbar \omega_{0}}{T}\right)\right]} \simeq \frac{\Sigma_{0}}{\tau_{0}^{\text {intra }}}\left[\left(\mathcal{N}_{0}+1\right) \exp \left(-\frac{\hbar \omega_{0}}{T}\right)-\mathcal{N}_{0}\right]
$$

where

$$
\frac{\Sigma_{0}}{\tau_{0}^{\text {intra }}}=2 G_{0}\left(\frac{T}{T_{0}}\right)^{3} \int_{0}^{\infty} \frac{d u u\left(u+\hbar \omega_{0} / T\right)}{\left[1+\exp \left(u-\varepsilon_{F} / T\right)\right]} .
$$

If $\left|\varepsilon_{F}\right|<T$, Eq. (A4) yields

$$
\frac{\Sigma_{0}}{\tau_{0}^{\text {intra }}} \simeq \frac{\pi^{2} G_{0}}{6}\left(\frac{T}{T_{0}}\right)^{2}\left(\frac{\hbar \omega_{0}+2.19 T}{T_{0}}\right) .
$$

Comparing Eqs. (A3) and (A6), for this case we obtain

$$
\eta_{0}=\frac{\tau_{0}^{\text {intra }}}{\tau_{0}^{\text {inter }}}=\frac{1}{\pi^{2}}\left(\frac{\hbar \omega_{0}}{T}\right)^{2}\left(1+2.19 \frac{T}{\hbar \omega_{0}}\right)^{-1} .
$$

If $\hbar \omega_{0}>\varepsilon_{F} \gg T$,

$$
\frac{\Sigma_{0}}{\tau_{0}^{\text {intra }}} \simeq G_{0} \frac{\hbar \omega_{0} \varepsilon_{F}^{2}\left(1+2 \varepsilon_{F} / 3 \hbar \omega_{0}\right)}{T_{0}^{3}} \simeq G_{0} \frac{\hbar \omega_{0} \varepsilon_{F}^{2}}{T_{0}^{3}},
$$

so that invoking Eq. (A3), we obtain

$$
\eta_{0} \simeq \frac{1}{6}\left(\frac{\hbar \omega_{0}}{\varepsilon_{F}}\right)^{2} .
$$

In a wide range of variations of $\varepsilon_{F}$ and $T$, one can use the following interpolation:

$$
\eta_{0}=\frac{\hbar^{2} \omega_{0}^{2}}{\left(6 \varepsilon_{F}^{2}+\pi^{2} T^{2}\right)} .
$$


[1] A. H. Castro Neto, F. Guinea, N. M. R. Peres, K. S. Novoselov, and A. K. Geim, Rev. Mod. Phys. 81, (2009).

[2] V. Ryzhii, M. Ryzhii, and T. Otsuji, J. Appl. Phys. 101, 083114 (2007).

[3] M.Ryzhii and V.Ryzhii, Jpn. J. Appl. Phys. (Express Lett.) 46, L151 (2007).

[4] F. Rana, IEEE Trans. Nanotechnol. 7,91 (2008).

[5] V.Ryzhii, M.Ryzhii, and T.Otsuji, Phys. Stat. Sol. (c) 5, 261 (2008).

[6] L. A. Falkovsky and A. A. Varlamov, Eur. Phys. J. B 56, 281 (2007).

[7] N. M. R. Peres, F. Guinea, and A. H. Castro Neto, Phys. Rev. B 73, 125411 (2006).

[8] M. Sprinkle, D. Suegel, Y. Hu, J. Hicks, A. Tejeda, A. Taleb-Ibrahimi, P. Le Fevre, F. Bertran, S. Vizzini, H. Enriquez, S. Chiang, P. Soukiassian, C. Berger, W. A. de Heer, A. Lanzara, and E. H. Conrad, Phys. Rev. Lett. 103, 226803 (2009).

[9] M. Orlita and M. Potemski, Semicond. Sci. Technol. 25, 063001 (2010).

[10] A. Dubinov, V. Ya. Aleskin, M. Ryzhii, T. Otsuji, and V. Ryzhii, Appl. Phys. Express 2, 092301 (2009).

[11] V. Ryzhii, M. Ryzhii, A. Satou, T. Otsuji, A. A. Dubinov, and V. Ya. Aleshkin, J. Appl. Phys. 106, 084507 (2009).

[12] V. Ryzhii, A. A. Dubinov, T. Otsuji, V. Mitin, and M. S. Shur, J. Appl. Phys. 107, 054505 (2010).

[13] F. T. Vasko and V. Ryzhii, Phys. Rev. B 77, 195433 (2008).

[14] A. Satou, F. T. Vasko, and V. Ryzhii, Phys. Rev. B 78, 115431 (2008).

[15] A. Satou, T. Otsuji, and V.Ryzhii, In. Techn. Dig. 2nd Int. Symp. on Graphene Devices: Technology, Physics, and Modeling, Sendai, Japan, Oct.27-29, 2010, p. 80; Jpn. J. Appl. Phys. (2011), in press.

[16] F. Rana, P. A. George, J. H. Strait, S. Shivaraman, M. Chanrashekhar, M. G. Spencer, Phys. Rev. B 79, 115447 (2009).

[17] R. S. Shishir, D. K. Ferry, and S. M. Goodnick, J.Phys.: Conf. Ser. 193, 02118 (2009).

[18] A. Javey, J. Guo, M. Paulsson, Q. Wang, D. Mann, M. Lundstrom, and H. Dai, Phys. Rev. Lett. 92, 106804 (2004).
[19] M. Lazzwei, S. Piscanec, F. Mauri, A. C. Ferrari, and J. Robertson, Phys. Rev. Lett. 95, 236802 (2005).

[20] E. Pop, D. Mann, J. Cao, Q. Wang, K. Goodson, and H. Dai1, Phys. Rev. Lett. 95, 155505 (2005).

[21] M. A. Kuroda and J.-P. Leburton, Appl. Phys. Lett. 89, 103102 (2006).

[22] C. Auer, F. Schurer, and C. Ertler, Phys. Rev. B 74, 165409 (2006).

[23] G. Pennigton, S. J. Kilpatrick, and A. E. Wickenden, Appl. Phys. Lett. 93, 093110 (2008)

[24] M. Steiner, M. Freitag, V. Perebeinos, J. C. Tsang, J. P. Small, M. Kinoshita, D. Yuan, J. Liu, and P. Avouris, Nature Nanotech. 4, 320 (2009).

[25] A. V. Kuznetsov and C. J. Stanton, Phys. Rev. Letts. 73, 3243 (1994).

[26] A. V. Kuznetsov and C. J. Stanton, Phys. Rev. B 51, 7555 (1995).

[27] J.-H. Kim, K.-J. Han, N.-J. Kim, K.-J. Yee, Y.-S. Lim, G. D. Sanders, C. J. Stanton, L. G. Booshehri, E. H. Hroz, and J. Kono, Phys. Rev. Lett. 102, 037402 (2009).

[28] M. Dyakonov and M. Shur, Phys. Rev. Lett. 71, 2465 (1993)

[29] M. Shur, Electron. Lett. 46 , S18 (2010).

[30] A.A. Balandin, S. Ghosh, D.L. Nika and E.P. Pokatilov, Fullerenes, Nanotubes and Carbon Nanostructures, 18, 474 (2010).

[31] P. A. George, J. Strait, J. Dawlaty, S. Shivaraman, M. Chandrashekhar, F. Rana, and M. G. Spencer, Nano Lett. 8, 4248 (2008).

[32] H. Wang, J. Strait,P. A. George, S. Shivaraman,V. B. Shields, M. Chandrashekhar, F. Rana,M. G. Spencer, C. S. Ruiz-Vargas, and J. Park, ArXiv 2009, 0909.4912.

[33] T. Elsaesser, In. Techn. Dig. 2nd Int. Symp. on Graphene Devices: Technology, Physics, and Modeling, Sendai, Japan, Oct.27-29, 2010, p. 48.

[34] P. Neugebauer, M. Orlita, C. Faugeras, A.-L. Barra, and M. Potemski, Phys. Rev. Lett. 103, 136403 (2009).

[35] L. A. Falkovsky, Phys. Rev. B 75, 03349 (2007).

[36] O. G. Balev, F. T. Vasko, and V. Ryzhii, Phys. Rev. B 79, 165432 (2009). 